\title{
WORKFORCE Physician associates advance patient safety
}

\author{
Authors: Laura Chenevert ${ }^{A}$ and Kate Bascombe ${ }^{B}$
}

\begin{abstract}
As the physician associate (PA) profession grows and awareness about the role increases, it is important to understand how PAs promote patient safety. In this article, we explore the current literature regarding the contribution of PAs to patient safety, considering their training, day-today clinical work and influence on recognised factors that affect patient safety.
\end{abstract}

KEYWORDS: patient safety, physician associate, patient experience, teamwork, physician assistant

DOI: $10.7861 /$ fhj.2021-0178

\section{Introduction}

Physician associates (PAs) are one of the newest members of the multidisciplinary team (MDT) in the UK. There are currently 1,788 PAs registered with the Faculty of PAs (FPA) at the Royal College of Physicians (RCP) practising in the UK. PAs were officially introduced to the UK in 2003 and are trained to medically assess, diagnose, treat and manage patients under the supervision of a doctor. The competencies and scope of practice for PAs are outlined in the Competence and Curriculum Framework (CCF) produced by the Department of Health in $2006 .{ }^{2}$

We present the evidence for PAs contributing to patient safety, considering their role and impact on core principles and markers of a safe clinical environment including training, continuing professional development (CPD), team dynamics, continuity, burnout and patient experience. We also highlight important aspects of the PA profession that require further research and investigation regarding patient safety.

\section{Training PAs}

To gain entry into a PA training programme in the UK, applicants need to have obtained an undergraduate degree in a life science at a 2:2 or higher, depending on the institution, to ensure an adequate academic foundation. Where resources allow, PA schools use multiple mini-interviews (MMIs) to ensure selection considers

Authors: Aphysician assistant, New York-Presbyterian/Columbia University Irving Medical Center, New York, USA; ${ }^{B}$ physician associate and senior lecturer, St George's, University of London, London, UK applicants' non-cognitive abilities. These are an observed structured clinical examination (OSCE) style interview used for healthcare professionals for more than 20 years, with a recognised and established evidence base. ${ }^{3}$

Selecting students with both the necessary academic aptitude and recognised attitudes to practise with integrity and empathy is the first step to ensuring graduation of a safe healthcare professional. ${ }^{3}$ With the General Medical Council (GMC) regulation of the profession expected at the end of 2022, there will also be accreditation of PA training programmes. This regulatory quality assurance is a fundamental step in ensuring the graduation of safe healthcare practitioners into the workforce. The GMC is working closely both with the FPA and training institutions to review the existing curriculum. It can be expected that, in line with the standards required of medical schools across the UK, patient safety will be a cornerstone of the requirements.

With this in mind, there will be over 2,000 PAs working in the UK that trained prior to the introduction of GMC guidance. Currently, the CCF outlines the core tenets of ensuring safe practitioners such as safeguarding and recognising working limitations and working within them. ${ }^{2}$ It identifies the recertification system (PAs are required to sit the qualifying written exam every 6 years) as a means of demonstrating that the PA remains safe to practise for the purpose of 'protecting the interest and safety of the patient.'2 PA students are also expected to demonstrate professional behaviour and probity, risk management, teamwork, and an understanding of ethical and legal issues.

With the current absence of national accreditation for PA training programmes, the national qualifying exam (both OSCE and written) ensures that there is a common framework and standard of skill that is met by all practising PAs. Patient safety is intrinsic in the assessment of therapeutic knowledge, communication and demonstrating an acknowledgement of one's own limitations.

As identified by Health Education England (HHE), embedding continued training and development is essential for patient safety. ${ }^{4}$ CPD requirements are set out by the FPA and aim to ensure practising PAs update, enhance and develop their knowledge and skills to ensure they are practising safely. ${ }^{5}$

\section{On the job}

According to a survey completed by organisational leaders of 27 academic medical centres across the USA, PAs and nurse practitioners (NPs) were valuable to their institutions in a number of different metrics. Top reasons for hiring PAs/NPs were resident (specialist trainee equivalent) hour restrictions, to improve patient 
throughput, to increase patient access, to improve patient safety and quality of care, to reduce length of stay, and to improve continuity of care. ${ }^{6}$

This is a consistent finding when looking at research of the PA role worldwide. A mixed-methods study reviewing the history and status of PAs in 15 nations through literature review, reports and interviews with healthcare stakeholders internationally found 'The utilization of PAs, particularly in primary healthcare roles, increases access to services, is cost-beneficial, and shows a physicianequivalent quality of care."

Timmermans et al performed a multicentre matched control study in the Netherlands comparing care delivered by a medical doctor (MD) only model versus an PA/MD model across 34 inpatient wards. ${ }^{8}$ Data on 2,307 patients followed for 1 month from admission to discharge showed no difference in indicators for quality and safety of care between MD and PA/MD teams. ${ }^{8}$

A study by Drennan et al used a mixed methods, multiple case study design to look at the contribution of PAs across six acute care hospitals in England through interviews, observations, work diaries and documentary analysis. ${ }^{9}$ Participants included 43 PAs, 77 other health professionals, 28 managers and 28 patients/relatives. The results showed that PAs were "acceptable, appropriate and safe members of the medical/surgical teams by the majority of doctors, managers and nurses. ${ }^{9}$

Drennan et al performed an observational study in general practice of 2,086 patient records who presented for same-day consultation across 12 different sites in England. They compared the outcomes and costs of same-day consultations between general practitioners (GPs) and PAs. There were no significant differences in rates of re-consultation, rates of diagnostic tests ordered, referrals or prescriptions issued between the GP and PA groups. $^{10}$

\section{Burnout}

The workload on healthcare providers is ever increasing, a large factor contributing to clinician burnout. A systematic review by Hall et al showed that poor wellbeing and moderate to high levels of burnout are associated with poor patient safety outcomes, such as medical errors." A systematic review by Garcia et al also showed a relationship between worsening patient safety and high levels of provider burnout. ${ }^{2}$ PAs can be part of the solution to ease the workload.

A study by Deis et al showed that PAs reduced the workload of general surgery residents in the USA, and improved care. PAs were found helpful in completing tasks such as discharge paperwork and answering pages. The presence of the PA on the team also allowed the resident to be in the operating room (OR) more and attend educational events. ${ }^{13}$ Similarly, a systematic review of 29 articles performed by Johal and Dodd demonstrated that 'resident workload decreased, sleep time increased, and operating time improved' with the addition of PAs and NPs to adult surgical and trauma services. ${ }^{14}$

\section{Other aspects of patient safety}

\section{Team dynamics}

Teamwork is essential to deliver safe care to patients. Ghadiri highlights the importance of individual professions (including PAs) understanding each other's roles and limitations to ensure a safe working environment. ${ }^{15}$ There is evidence that improved teamwork in healthcare is linked to reduced patient complications and death. ${ }^{16}$ Studies that look at incidents or adverse events show that flawed teamwork is often responsible rather than a clinical skills deficiency. ${ }^{17}$

Strong working relationships between team members is vital and is built over time. This includes having strong communication skills and working well with each other. PAs are permanent members of the team and have the potential to develop strong working relationships with the MDT, including consultants and nurses. A PA is available to answer clinical questions from nurses and ancillary members of the team. One part of Moote et al's research on the value of advanced practice providers (APPs) was a survey. They found the highest rated statement on the survey to be that APPs are "easily accessible to other members of the health care team to discuss and provide direction for patient care. ${ }^{6}$

The introduction of PAs to the UK, understandably, brought with it a concern for the impact of the role on postgraduate training in medicine and surgery. However, a mixed methods explorative study across five sites in the north of England demonstrated that, within 6 months of PAs joining the team, a third of junior doctors thought that their training had been 'enhanced'. Half of junior doctors thought there had been 'no overall impact'.18

\section{Continuity of care}

There is a significant association between increased continuity and decreased health utilisation (including hospitalisation and emergency visits). In addition, patient satisfaction improved with increased continuity. ${ }^{19}$ A systematic review showed that increased continuity of care by doctors is associated with lower mortality rates. ${ }^{20}$

Van Vught et al performed semi-structured interviews with 65 supervising medical specialists in the Netherlands. They collected data in the first year and five years after PA employment. They found that the "primary motive for employing a PA in Dutch healthcare is to increase continuity and quality of care' and this was consistent with the outcomes of their study. ${ }^{21}$

\section{Patient experience}

In order to provide safe, high-quality care, patients and clinicians need a relationship based on trust, respect and strong communication. Patients should be encouraged to be advocates of their own care and actively participate in their healthcare decisions. Providers must create an environment and build a relationship with patients that fosters these dynamics.

A qualitative study completed by Taylor et al showed that PAs demonstrated successful patient-clinician communication in acute hospital encounters. They interviewed 15 patients and patient representatives in five different hospitals in England. Four interrelated communication experiences were important to participants who were satisfied with the encounter (with the PA) in general: feeling trust and confidence in the relationship, sharing relevant and meaningful information, experiencing emotional care and support, and sharing discussion on illness management and treatment. ${ }^{22}$

Meijer et al looked at patient satisfaction with Dutch PAs compared with GPs by surveying patients who received out-ofoffice-hours care at four different GP practices. They collected patient satisfaction data from 92 patients seen by a GP and 100 patients seen by a PA. They found no statistically significant 
difference in patient satisfaction between care provided by a GP versus a PA. ${ }^{23}$

Patients are largely satisfied with the care they receive from PAs and accepting of the PA role. ${ }^{24}$ Overall, patients appear to be satisfied with their care once their needs are met, regardless of who actually provides the care. ${ }^{23,24}$ The PA can be an additional resource for answering patients' questions, addressing concerns and coordinating their care.

\section{Conclusion and future directions}

PAs working globally are achieving the benchmarks of safe patient care. This is due to PAs' rigorous training and professional standards together with the utility and value of the PA role in dayto-day clinical practice. PAs are important members of the clinical team contributing to continuity of care and meeting patients' expectations in providing quality care.

With continued recognition and utilisation of the PA profession in the UK, it will be increasingly important to assess and evaluate their contribution to patient safety. So far, there is evidence that PAs have a synergistic relationship with their MDT in working towards the highest levels of patient care. We suggest that future research should look at the whole professional journey from student selection and education through to their delivery, working relationships and patient satisfaction both in primary and secondary care.

\section{Conflicts of interest}

Laura Chenevert and Kate Bascombe are members of the editorial board for Future Healthcare Journal.

\section{References}

1 Faculty of Physician Associates. Focus on physician associates: census 2020. FPA, 2020. www.fparcp.co.uk/about-fpa/fpa-census [Accessed 23 September 2021].

2 Department of Health. Competence and Curriculum Framework for the Physician Assistant. Faculty of Physician Associates, 2012. www.fparcp.co.uk/professional-development/fpa-materials

3 Ali S, Sadiq Hashmi MS, Umair M, Beg MA, Huda N. Multiple mini-interviews: current perspectives on utility and limitations. Adv Med Educ Pract 2019:10:1031-8.

4 Health Education England. Improving Safety Through Education and Training. HEE, 2016. www.hee.nhs.uk/sites/default/files/ documents/Improving \% 20safety \% 20through \% 20education $\% 20$ and $\%$ 20training.pdf

5 Faculty of Physician Associates. Continuing professional development (CPD). FPA. www.fparcp.co.uk/professional-development/cpd

6 Moote M, Krsek C, Kleinpell R, Todd B. Physician assistant and nurse practitioner utilization in academic medical centers. Am J Med Qual 2011;26:452-60.

7 Cawley JF, Hooker RS. Determinants of the physician assistant/ associate concept in global health systems. Int J Healthc 2018;4:50.
8 Timmermans MJC, van Vught AJAH, Peters YAS et al. The impact of the implementation of physician assistants in inpatient care: A multicenter matched-controlled study. PLoS One 2017;12:e0178212

9 Drennan VM, Halter M, Wheeler C et al. What is the contribution of physician associates in hospital care in England? A mixed methods, multiple case study. BMJ Open 2019;9:e027012.

10 Drennan VM, Halter M, Joly L et al. Physician associates and GPs in primary care: a comparison. Br J Gen Pract 2015;65:e344-50.

11 Hall LH, Johnson J, Watt I, Tsipa A, O'Connor DB. Healthcare staff wellbeing, burnout, and patient safety: a systematic review. PLoS One 2016;11:e0159015.

12 Garcia CL, Abreu LC, Ramos JLS et al. Influence of burnout on patient safety: systematic review and meta-analysis. Medicina (Kaunas) 2019;55:553.

13 Dies N, Rashid S, Shandling M et al. Physician assistants reduce resident workload and improve care in an academic surgical setting. JAAPA 2016;29:41-6

14 Johal J, Dodd A. Physician extenders on surgical services: a systematic review. Can J Surg 2017;60:172-8.

15 Ghadiri SJ. Physician associates: an asset for physician training and a 21st-century NHS? FHJ 2020;7:e9-10

16 Weller J, Boyd M, Cumin D. Teams, tribes and patient safety: overcoming barriers to effective teamwork in healthcare. Postgrad Med J 2014:90:149-54.

17 Manser T. Teamwork and patient safety in dynamic domains of healthcare: a review of the literature. Acta Anaesthesiol Scand 2009;53:143-51.

18 Roberts S, Howarth S, Millott H, Stroud L. Experience of the impact of physician associates on postgraduate medical training: A mixed methods -exploratory study. Clin Med 2019;19:4-10.

19 Van Walraven C, Oake N, Jennings A, Forster AJ. The association between continuity of care and outcomes: a systematic and critical review. Journal of Evaluation in Clinical Practice 2010;16:947-56.

20 Pereira Gray DJ, Sidaway-Lee K, White E et al. Continuity of care with doctors - a matter of life and death? A systematic review of continuity of care and mortality. BMJ Open 2018:8:e021161.

21 van Vught AJAH, van den Brink GTWJ, Wobbes T. Implementation of the Physician assistant in Dutch health care organizations. The Health Care Manager 2014;33:149-53.

22 Taylor F, Halter M, Drennan VM. Understanding patients' satisfaction with physician assistant/associate encounters through communication experiences: a qualitative study in acute hospitals in England. BMC Health Serv Res 2019;19:603.

23 Meijer K, Kuilman L. Patient satisfaction with PAs in the Netherlands. JAAPA 2017;30:1-6.

24 Hooker RS, Moloney-Johns AJ, McFarland MM. Patient satisfaction with physician assistant/associate care: an international scoping review. Hum Resour Health 2019;17:104

Address for correspondence: Laura Chenevert, Department of Emergency Medicine, New York-Presbyterian/Columbia University Irving Medical Center, 622 West 168th Street Suite VC-260, New York, New York 10032-3784, USA.

Email: Imc2274@cumc.columbia.edu

Twitter: @LauraChenevert; @ksbascombe 